\title{
Research on Risk Assessment Model of Industrial Park Earthquake Disaster Chain
}

\author{
Danxiang $\mathrm{Ma}^{1,2, a}$, Jianyu Chu ${ }^{1,2, b}$, Lingli Chen ${ }^{1,2, c}$ \\ ${ }^{1}$ College of Civil And Architectural Engineering, Hebei United University, Tangshan 063009, China \\ ${ }^{2}$ Earthquake Engineering Rresearch Center of Hebei Province, Tangshan 063009, China \\ aheiseyouhuo@126.com, btschujy@126.com, ${ }^{\mathrm{c}} 729560707 @ q q . c o m$
}

Keywords: Earthquake; Disaster Chain; Risk Assessment

\begin{abstract}
The factors influencing disaster chain risk were analyzed, and the risk assessment model of earthquake disaster chain was established. The methods to determine relevant parameters in the model were given. An example that analyzed the risk of earthquake-fire disaster chain in a petrochemical system was presented to illustrate the applicability and effectiveness of the model. This provides a basis to prevent and control the earthquake disaster chain risk of industrial park.
\end{abstract}

\section{Introduction}

There are many dangerous sources in the industrial park, the disasters have the characteristics of variety and concentration, and the development of the industrial park's disasters is complicated and chained. When another kind of disaster was caused by earthquake, a chained and complicated disaster effect was forming. Its destructiveness often exceeds the earthquake damage. The Japanese 3.11 earthquake triggered a series of secondary disasters such as the tsunami, nuclear leak, fire, explosion, etc. The human and financial tolls caused by these disasters far exceed the direct loss of earthquake, and the unexpected difficulties are brought for the emergency rescue.

At present, there is a deficiency of the research on risk assessment of earthquake disaster chain. Li Tianqi studied the forming process and mechanism of the earthquake disaster chain, and explained the methods of shutting off and controlling of the disaster chain [1]. Yu Shizhou took "earthquake $\rightarrow$ fire" for example, analyzed the mechanism of the earthquake disaster chain, established physical model of earthquake disaster chain, gave quantitative probability model, and suggested the measures of chain-cutting [2]. Wang Xiang put forward risk assessment model of the regional disaster chain basing on the probability of a disaster cause another, the loss of an event on a disaster chain and edge's vulnerability [3]. Zhang Weixing proposed risk assessment concept model of disaster chain by combining Wenchuan earthquake disaster chain [4].

On the basis of existing research results, this paper established risk assessment model of industrial park earthquake disaster chain. With "earthquake $\rightarrow$ fire" disaster chain of the petrochemical system, for example, the effectiveness of the model was validated. The public safety assessment method was provided to prevent industrial park earthquake and secondary disasters.

\section{Analysis of Factor Influence for Disaster Chain}

The concept of disaster chain was first put forward in 1987 by Guo Zengjian that disaster chain was a phenomenon that the series of disasters continuously occurred [5]. Shi Peijun defined disaster chain as a series of disaster phenomena caused by a disaster-causing factors or ecological environment changes, and divided it into series disaster chain and concurrency disaster chain [6].

Natural disaster risk is the expected loss of life, property and economy caused by particular natural disasters in a certain area and within a given time period [7]. It can be analyzed from three aspects: the hazards of disaster, the supporting body of disaster risk and the vulnerability of supporting body.

The measurement of disaster risk mainly relates to the intensity of risk source and the probability of disaster. In general, the greater the intensity of risk source is, the greater the probability of disaster 
is, and disaster risk is higher. Risk source doesn't mean risk, and there is the loss of life and economy. Therefore, the supporting body of disaster risk must be analyzed. Although the reasons and kinds of the disaster are different, the losses are also different, but the supporting body should consist of three factors that were person, substance and environment. The vulnerability of supporting body is that under a background of social, politics, economy and culture, the specific disaster-supporting body shows the nature that is more vulnerable for some disaster in a pregnant disaster environment's area. It is generally accepted that the greater vulnerability more leads to disaster, on the contrary, and the smaller the vulnerability doesn't more easily form the disaster. Therefore, the risk of earthquake disaster chain should be analyzed from four aspects that are the intensity of risk source, the probability of disaster, the supporting body of disaster risk and the vulnerability of supporting body.

\section{Risk Assessment Model of Disaster Chain}

Risk Assessment Model. In this article, grid partition of assessment units was adopted to determine the basic unit of risk assessment. Eq. 1 is the description of earthquake disaster chain risk in given area from above four aspects.

$$
R_{j}=\left(w_{1} \times H_{j}, w_{2} \times E_{j}, w_{3} \times M_{j}\right) \bullet \sum_{i=1}^{N} P_{i} \times\left(v_{i j 1}\left(m_{i}\right), v_{i j 2}\left(m_{i}\right), v_{i j 3}\left(m_{i}\right)\right)
$$

In this equation, $R$ represents the comprehensive disaster risk of grid $j . H_{j}, E_{j}$ and $M_{j}$ respectively represent the dimensionless value of person, substance and environment. $w_{1}, w_{2}$ and $w_{3}$ represent the vulnerability weight of person, substance and environment. $N$ is the total number of disaster in the disaster chain. $P_{i}$ is the probability of disaster $i$. Among them, and $P_{1}$ is the probability of risk source. $m_{i}$ is the intensity of risk source $i$. When the intensity of risk source $i$ is $m_{i}, v_{i j 1}\left(m_{i}\right) 、 v_{i j 2}\left(m_{i}\right)$ and $v_{i j 3}\left(m_{i}\right)$ represent he vulnerability of supporting body(person, substance and environment).

Determination of Parameters. 1) Dimensionless method of supporting body and the determination of the weights.

Because the physical units of supporting body are not the same, the dimensionless method must be applied to the data. [8] exploited the coverage area of environment and buildings to non-dimensionally analyze them. In this paper, we improve the dimensionless method and exploit the appraisal of environment and buildings. In this way, the result will be more accurate, and environment and buildings needn't be classified. The method is shown below:

1) The dimensionless value of person $H_{j}$

$$
H_{j}=\frac{n_{j}}{N}
$$

In this equation, $n_{j}$ is the number of people in grid $j$, and $N$ is the total number of the study area.

2) The dimensionless value of substance $M_{j}$

$$
M_{j}=\frac{a_{j}}{A}
$$

$a_{j}$ is the appraisal of buildings and structures in grid $j$, and $A$ is the total appraisal of all buildings and structures in the study area.

3) The dimensionless value of environment $E_{j}$

$$
E_{j}=\frac{b_{j}}{B}
$$

$b_{j}$ is the appraisal of environment in grid $j$, and $B$ is the total appraisal of all environmental in the study area. 
The weight of supporting body may be determined by the analytic hierarchy process. According to the conclusion of ARAMIS, person nearly accounts for $75 \%$, and environment and substance nearly account for $25 \%$ and $5 \%$ of the overall vulnerability [8].

2) Probability of earthquake and other disasters in chain.

Earthquake is the source in earthquake disaster chain, the probability can be computed by probabilistic seismic hazard analysis method [9], and it is the major advantage that the method considers the irregular distributive characters of the seismic activity. In a disaster chain, the probability of other disasters but the source is the probability of a disaster caused by the previous disaster, and it is different from the single probability of the disaster. [3,4] used the co-occurrence probability of two disasters to express, and it was estimated by Jaccard index. In the industrial park, calculating the probabilities of fire, explosion, toxic gas diffusion and other disasters caused by earthquake is more appropriate by the fault tree analytical method. The equations are shown as the following [8].

$$
\begin{aligned}
& P_{e}=1-\left(1-q_{1}\right)\left(1-q_{2}\right) \cdots\left(1-q_{m}\right)=1-\prod_{i=1}^{m}\left(1-q_{i}\right) \\
& P_{e}=q_{1} q_{2} \cdots q_{m}=\prod_{i=1}^{m} q_{i}
\end{aligned}
$$

Above the two equations, $P_{e}$ is the probability of output event $e, q_{i}$ is the probability of the input event $i$, and $\mathrm{m}$ is the quantity of input events in a logic gate. When there is "or" relationship between the input events, Eq. 5 is used. When there is "and" relationship between the input events, Eq. 6 is used. In "earthquake $\rightarrow$ fire" disaster chain of a petrochemical system, pipeline damage, storage container damage and structural collapse that lead to a leakage of inflammable material leakage are "or" relationship, as shown in Figure 1. The leakage and fire source that cause an explosion are "and" relationship.

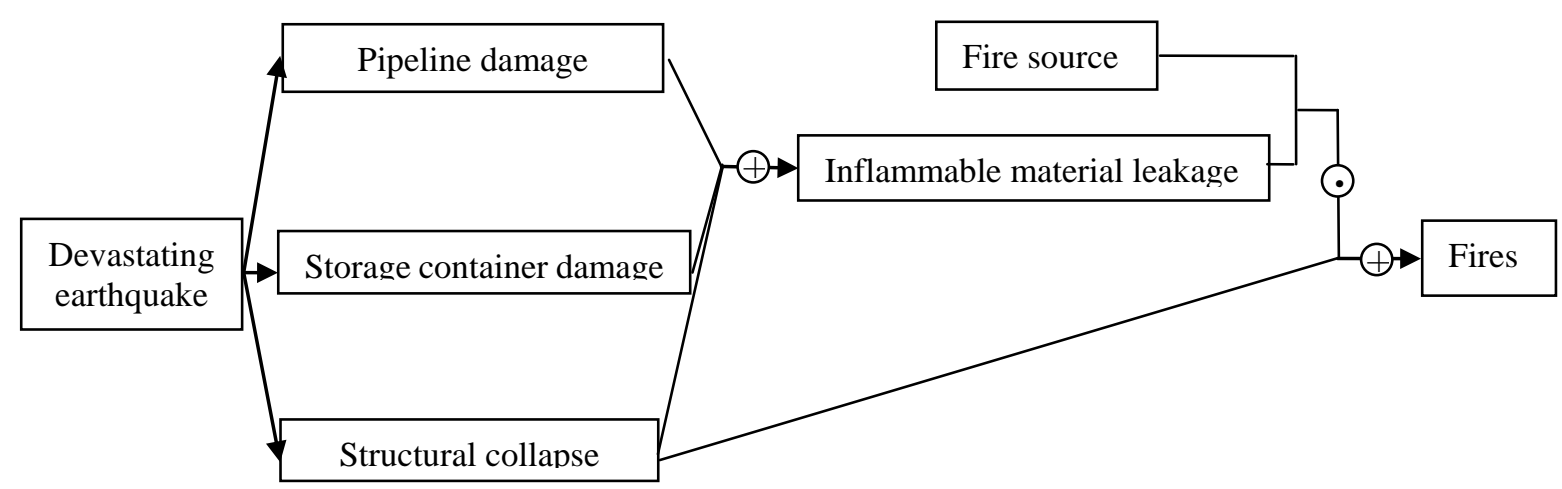

Fig. 1 Earthquake $\rightarrow$ fire Disaster

For petrochemical system, the other nodes in earthquake disaster chain mainly involve pipeline damage, storage container damage and structural collapse. Because of many factors, it is difficult to accurately determine their probabilities. For example, storage container damage not only is related to physical effect of the earthquake, but also has relation with the material, position, etc of storage container. There are usually four methods: the probability function method, empirical data, finite element method, and expert scoring method.

3) Analysis of supporting body vulnerability.

For a single disaster, the analytical methods of supporting body vulnerability mainly include determining the regional vulnerability according to historical data, the regional vulnerability assessment based on an index system and an actual survey [10]. There are delays for the occurrence of disasters in the disaster chain, and there are overlaps in the impact area. However, the sum of the 
same supporting body vulnerability should be less than or equal to 1 for a grid. Eq. 7 must be satisfied.

$$
\sum_{i=1}^{N} V_{i j} \leq 1
$$

In this equation, $N$ is the total number of the disasters in disaster chain. $V_{i j}$ is the same supporting body vulnerability in grid $j$, when disaster $i$ happens.

\section{Analysis and Application of Instance}

In North China, an industrial park is the concentrated area of steel, petrifaction, power, port and manufacture. Petrochemical industries are located in the southeast of industrial area, the planning area is 27.8 square kilometers, and the large amounts of methane is produced, stored, used, managed, transported, and disposed. Once the earthquake struck, it easily led to secondary disasters such as the leakage of dangerous chemical products, fires, explosions. Disaster chain would be formed that would amplify the impacted area and the degree of damage and loss.

There are 8 methane storage tanks in analyzed area of the instance, and not farms, nature reserves and other supporting body. Therefore, person and substance will only be analyzed. First, population density of the area must be counted, and assess the value of buildings and structures. According to AHP, the weights of person and substance are 0.936 and 0.064 respectively [8]. Through probabilistic seismic hazard analysis method, the horizontal peak ground acceleration is $125.3 \mathrm{gal}$, considering the exceeding probability to be 10 percent in the future 50 years. According to historical data, the probability of a medium above damage to buildings and structures is $55 \%$. The human mortality in buildings is $2.85 \%$, based on the prediction model in [11] refs. Through expert evaluation, the conditional probability of the fires caused by earthquakes is $21.7 \%$. Then, the probability of earthquakes and fires is $2.17 \%$. According to jet fire calculation model and the damage probability equation of thermal radiation to human body [8], flame length, the relationship of thermal radiation distance and human mortality (exposure time was 30 seconds) and the destroying radius to buildings might be calculated. The comprehensive risk analysis result of the area is shown in Figure 2. The deeper the color, the greater the risk of "earthquake $\rightarrow$ fire" chain.

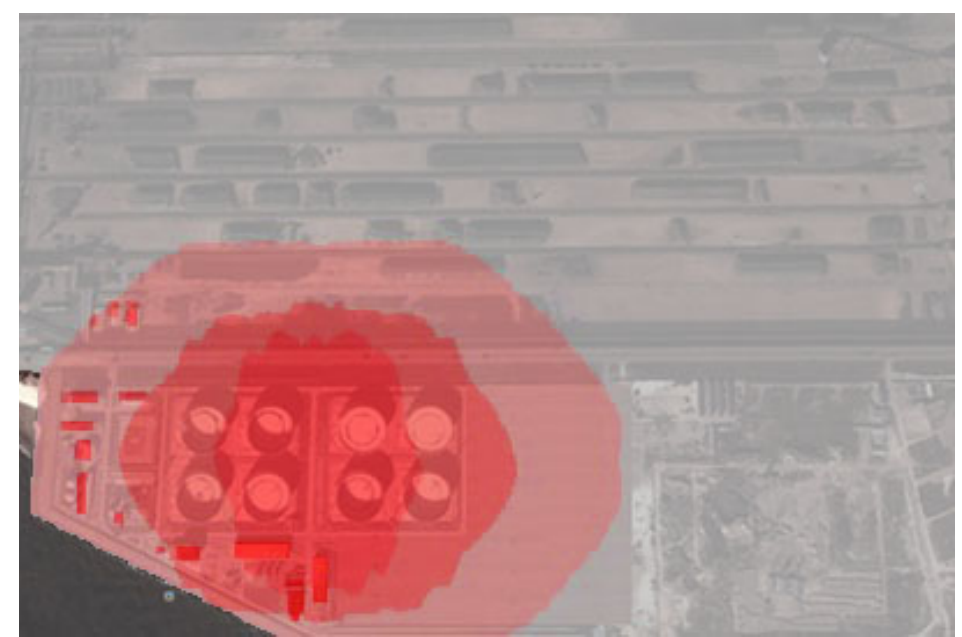

Fig. 2 Risk Analysis of Earthquake-fire Disaster Chain

\section{Conclusion}

At present, there are few quantitative research achievements about risk assessment of earthquake disaster chain. Considering the complexity and chain of disaster development, this paper describes the risk of earthquake disaster chain from four aspects that are the intensity of risk source, the 
probability of disaster, the supporting body of disaster risk and the vulnerability of supporting body. The risk assessment model of disaster chain that enriches quantitative analysis method is established. The probability of a disaster caused by the previous disaster is calculated by using the fault tree analytical method that divides input events into "and" and "or" relationship, so it is closer to secondary and transformational rule of earthquake disaster chain. The method that nondimensionalizes substance and environment by evaluation is proposed, and it makes the results more accurate. Finally, combining with an example of "earthquake $\rightarrow$ fire" disaster chain, the validity of the model is verified. The paper provides a public safety assessment method to prevent the earthquake and the secondary disasters in industrial park.

\section{Acknowledgements}

The authors gratefully acknowledge the funds provided by the national science-technology support program of China (No. 2013BAJ10B09-2) and the natural science foundation of Hebei province (No. E2013209262) for the work presented in this paper.

\section{References}

[1] T.Q. Li and Z.D. Zhao: Journal of Natural Disasters, Vol. 15 (2006), p. 148. (In Chinese)

[2] S.Z. Yu, L.X. Zhang, Z.D. Zhao and J.R. Zhong: China Civil Engineering Journal, Vol. 43 (2010), p. 479. (In Chinese)

[3] X. Wang: Research on Risk Assessment of Regional Disaster Chain (MS., Dalian University of Technology, China 2011). (In Chinese)

[4] W.X. Zhang and H.J. Zhou: Progress in Geography, Vol. 32 (2013), p. 130. (In Chinese)

[5] Z.J. Guo and B.Y. Qin: Journal of Catastrophology, Vol. 2 (1987), p. 25. (In Chinese)

[6] P.J Shi: Journal of Nanjing University (Natural Sciences), Special Issue on Research of Natural Disasters (1991), p. 37. (In Chinese)

[7] Q.Z. Lu, J.B. Peng and F.S. Zhao: Journal of Catastrophology, Vol. 18 2003, p 59. (In Chinese)

[8] M. Liu: Accident Risk Analysis Theory and Methodology (Peking University Press, China 2011). (In Chinese)

[9] PRC Standard: Evaluation of Seismic Safety Engineering Sites, GB17741-2005. (In Chinese)

[10] Y. Shi, S.Y. Xu, C. Shi, A.L. Sun and Q.L. Zhao: Journal of Natural Disasters, Vol. 20 (2011), p. 131. (In Chinese)

[11] Z.Q, Yin: Earthquke Engineering and Engineering Vibration, Vol. 11 (1991), p. 87. (In Chinese) 